\title{
Spectral CT Analysis of Solitary Pulmonary Nodules for Differentiating Malignancy from Benignancy: The Value of Iodine Concentration Spatial Distribution Difference
}

\author{
Linyu Wu, Guoquan Cao, Liang Zhao, Kun Tang, Jie Lin, Shouliang Miao, Tingting Lin, \\ Jieke Sun, and Xiangwu Zheng iD \\ Department of Radiology, The First Affiliated Hospital of Wenzhou Medical University, Wenzhou 325035, China \\ Correspondence should be addressed to Xiangwu Zheng; zxwu111@sina.com
}

Received 25 June 2018; Revised 23 September 2018; Accepted 21 November 2018; Published 9 December 2018

Academic Editor: Joanna Domagala-Kulawik

Copyright (C) 2018 Linyu Wu et al. This is an open access article distributed under the Creative Commons Attribution License, which permits unrestricted use, distribution, and reproduction in any medium, provided the original work is properly cited.

\begin{abstract}
Objective. The objective is to assess the value of spatial distribution difference in iodine concentration between malignant and benign solitary pulmonary nodules (SPNs) by analyzing multiple parameters of spectral CT. Methods. Sixty patients with 39 malignant nodules and 21 benign nodules underwent chest contrast CT scans using spectral imaging mode during pulmonary arterial phase (PP), arterial phase (AP), and venous phase (VP). Iodine concentrations of proximal and distal regions in pulmonary nodules on iodine-based material decomposition images were recorded. Normalized iodine concentration (NIC) and the differences in NIC between the proximal and the distal regions (dNIC) were calculated. The two-sample t-test and Mann-Whitney U-test were performed to compare the multiple parameters generated from spectral CT between malignant and benign nodules. Receiver operating characteristic (ROC) curves were generated to calculate sensitivity and specificity. Results. NIC in the proximal region $\left(\mathrm{NIC}_{\text {pro }}\right)$ and NIC in the distal region $\left(\mathrm{NIC}_{\text {dis }}\right)$ between malignant and benign nodules at $\mathrm{AP}\left(\mathrm{NIC}_{\text {pro }}, \mathrm{P}=0.012 ; \mathrm{NIC}_{\text {dis }}, \mathrm{P}=0.024\right)$, and $\mathrm{VP}\left(\mathrm{NIC}_{\text {pro }}, \mathrm{P}=0.005 ; \mathrm{NIC}_{\text {dis }}, \mathrm{P}=0.004\right)$ were significantly different. $\mathrm{NIC}_{\text {pro }}$ at $\mathrm{PP}(\mathrm{P}=0.037)$ was also found significantly different between malignant and benign nodules; however, no significant differences were found in $\mathrm{NIC}_{\mathrm{dis}}$ at $\mathrm{PP}(\mathrm{P}=0.093)$. In addition, the dNIC of malignant nodules was significantly higher than that of benign ones at PP (median and interquartiles $(0.31,0.11,0.57$ versus $-0.26,-0.5,-0.1) ; \mathrm{p} \leq 0.001$ ), AP (mean dNIC, $0.093 \pm 0.094$ versus $-0.075 \pm 0.060 ; \mathrm{p} \leq 0.001$ ), and VP (mean dNIC, $0.171 \pm 0.137$ versus $-0.183 \pm 0.127 ; \mathrm{p} \leq 0.001)$. The sensitivity and specificity $(93 \%, 95 \%$, respectively) of dNIC during VP were higher than other parameters, with a threshold value of -0.07 . Conclusions. Spectral CT imaging with multiple parameters such as $\mathrm{NIC}_{\mathrm{pro}}, \mathrm{NIC}_{\mathrm{dis}}$, and dNIC may be a new method for differentiating malignant SPNs from benign ones.
\end{abstract}

\section{Introduction}

Solitary pulmonary nodules (SPNs) which are defined as the isolated, round, or oval areas of increased opacity less than or equal to $3 \mathrm{~cm}$ in diameter, surrounded by lung parenchyma, should not be associated with atelectasis, pulmonary hilar enlargement, or pleural effusion [1-3]. The diagnosis and management of solitary pulmonary nodule are a common and costly challenge in medicine $[4,5]$. Conventional CT plays an important role in capturing morphological and enhancement features of SPNs. However, there is considerable overlap in the features between malignant and benign SPNs that lead to an erroneous diagnosis of pulmonary nodules $[6,7]$. Therefore, research is being directed towards the development of noninvasive functional imaging techniques, such as dual-energy spectral CT $[8,9]$.

Gemstone spectral imaging (GSI) is a novel introduced technique based on the rapid switching between high- and low-energy data sets from view to view during a single rotation on the high-definition GE Discovery CT750 HD scanner, which provides more analyzing tools and quantitative parameters to help distinguish the malignant SPNs from the benign ones $[8,10]$. It could enable the generation of material decomposition images that allows one to measure iodine component on iodine-enhanced images, and this is considered to be comparable to the real value of enhancement. Iodine concentration on iodine-enhanced images generated from spectral CT reflects the blood supply 
of SPNs, which is an essential evidence for differential diagnosis [11-14]. The spectral CT had multiple clinical uses in diagnosing pulmonary nodules, pulmonary embolism, assessing noninvasively angiogenesis of advanced gastric cancer, and differentiating small hepatocellular carcinoma from small hepatic hemangioma [13-17].

According to the previous research, Zhao et al. had firstly reported a new phenomenon of SPN heterogeneity in $\mathrm{PET} / \mathrm{CT}$, indicating that there was significant difference in spatial distribution of ${ }^{18}$ F-FDG metabolism between malignant and benign pulmonary nodules [18-21]. It has been reported that there was correlation between the SUV on $\mathrm{PET} / \mathrm{CT}$ imaging and the iodine concentration on spectral CT imaging [22], in reflecting the blood supply and vascular density of SPNs. As a follow-up research, we hypothesized that a similar phenomenon of SPN would be observed on the spectral CT, as on the PET/CT. To our best knowledge, no clinical studies have directly analyzed the iodine concentration spatial distribution difference of SPN using spectral CT to differentiate malignancy and benignancy. Therefore, the purpose of this study was to explore the predictive value of spatial distribution difference in iodine concentration in SPNs with spectral CT.

\section{Materials and Methods}

The local institutional review board approved this prospective study, and written informed consent was obtained from all patients (no. 2017-152).

2.1. Patients. From July 2015 to June 2016, a total of 68 consecutive patients with suspected solitary pulmonary nodules were recruited, which were identified on CT scans obtained at local hospitals and/or clinics.

The patients who were selected for this study met the following criteria: (a) the presence of solid SPNs with the diameter more than $8 \mathrm{~mm}$ and less than $30 \mathrm{~mm}$, (b) no contraindications to the administration of iodinated contrast material, and (c) having the ability to participate in the procedures cooperatively. Diameter was defined as the maximum diameter on the conventional thin-section CT scan with a lung window setting. The patients with ground glass nodules and/or nonsolid nodules were excluded on conventional thin-section CT scans. Ten patients were subsequently excluded: nine patients were excluded owing to the absence of pathologic diagnosis; one patient was excluded owing to noncooperation with the procedures. In the study, the followup CT of one patient was performed for 4 months, and the follow-up CT of other patient was performed for 6 months. So they were excluded from our research. As we known, if a nodule demonstrates a stable size for more than 2 years at comparison with prior radiographs, it has a high likelihood of being benign, and no further assessment is recommended [23]. In our study, a nodule demonstrates a stable size for more than 2 years at comparison with prior radiographs $(n=1)$; two nodules of the patient were disappeared after antiinflammatory therapy $(\mathrm{n}=2)$.
Thus, 60 consecutive patients in total (age range, 36-84 years; mean age, $61.4 \pm 10.3$ years, 40 men and 20 women) were included to this study. Sixty SPNs (mean diameter $18.03 \pm 5.77 \mathrm{~mm}$; range, $8-30 \mathrm{~mm}$ ) were eventually included in the data analysis. All the patients underwent spectral CT or tracheal-bronchial biopsy (transbronchial or percutaneous) or bronchoalveolar lavage or microbiological examination or nodule resection by video-assisted thoracic surgery or conventional follow-up CT. For all nodules, final diagnoses were subsequently confirmed by a video-assisted thoracic surgery $(\mathrm{n}=36)$, CT-guided percutaneous or transbronchial biopsy $(n=19)$, bronchoalveolar lavage $(n=2)$, and clinically follow-up CT $(n=3)$.

2.2. Groups. The SPNs were divided into two groups based on the final diagnosis. The malignant group $(n=39$, mean diameter, $19.26 \pm 5.99 \mathrm{~mm}$; range, $9-30 \mathrm{~mm}$ ) was composed of adenocarcinoma $(n=33)$, squamous cell carcinoma $(n=3)$, small cell carcinoma $(\mathrm{n}=1)$, and metastatic lung tumors $(\mathrm{n}=2)$. The benign group $(n=21$, mean diameter, $15.76 \pm 4.85 \mathrm{~mm}$; range, $8-28 \mathrm{~mm})$, included hamartoma $(\mathrm{n}=4)$, granuloma $(n=2)$, tuberculoma $(n=2)$, organizing pneumonia $(n=11$, comprising 10 organizing pneumonias confirmed with histological examination and 1 organizing pneumonia with their size unchanged for more than 2 year), and pulmonary inflammation (disappeared after anti-inflammatory therapy, $\mathrm{n}=2$ ).

2.3. Spectral CT Scans. Three-phase contrast-enhanced chest scans were performed on a spectral CT scanner (GE Discovery CT750HD HDCT; GE Healthcare, Milwaukee, WI, USA) using GSI mode and low dose protocols in all the patients. Spectral imaging was obtained at $15 \mathrm{~s}$ (pulmonary artery phase, PP), $30 \mathrm{~s}$ (artery phase, AP), and $60 \mathrm{~s}$ (venous phase, VP) after contrast medium injection, respectively. The nonionic contrast media Ioversol Injection $(320 \mathrm{mg} \mathrm{I} / \mathrm{ml}$, GE Healthcare) at the dose of $1.1 \mathrm{ml} / \mathrm{kg}$ was injected by using a pressure injector at a rate of $3.0 \mathrm{ml} / \mathrm{s}$ with antecubital venous access. Then, it was followed by $20 \mathrm{ml}$ saline at the same rate. The scanning parameters included dual $\mathrm{kVp}$ (80 and $140 \mathrm{kVp}$ ); tube current $275 \mathrm{~mA}$; scan range from apex to base of the lung; helical pitch 1.375:1; rotation speed of 0.7 $\mathrm{s}$; detector coverage of $40 \mathrm{~mm}$; field-of-view $(\mathrm{FOV}) 36 \mathrm{~cm}$; section thickness of $0.625 \mathrm{~mm}$; reconstruction interval of $0.625 \mathrm{~mm}$. The CT dose index volume was a value of 7.64 $\mathrm{mGy}$ for each phase with spectral CT acquisition (comparable to $7.48 \pm 1.22 \mathrm{mGy}$ dose administered for each phase of conventional contrast-enhanced scanning in a normal-size patient) at our institution.

2.4. Image Analysis. All the raw data were automatically reconstructed into images with slice thickness of 0.625 $\mathrm{mm}$ and spacing of $0.625 \mathrm{~mm}$, and then the images were transferred to an AW4.6 workstation (GE Volume Share 6 AW4.6; GE Healthcare) for analysis with the use of GSI Volume Viewer software package. Iodine-based and waterbased material decomposition images were obtained. Blinded to patient information and pathologic diagnosis, two radiologists (K.T. and L.Y.W, with 11 and 5 years of experience in chest 


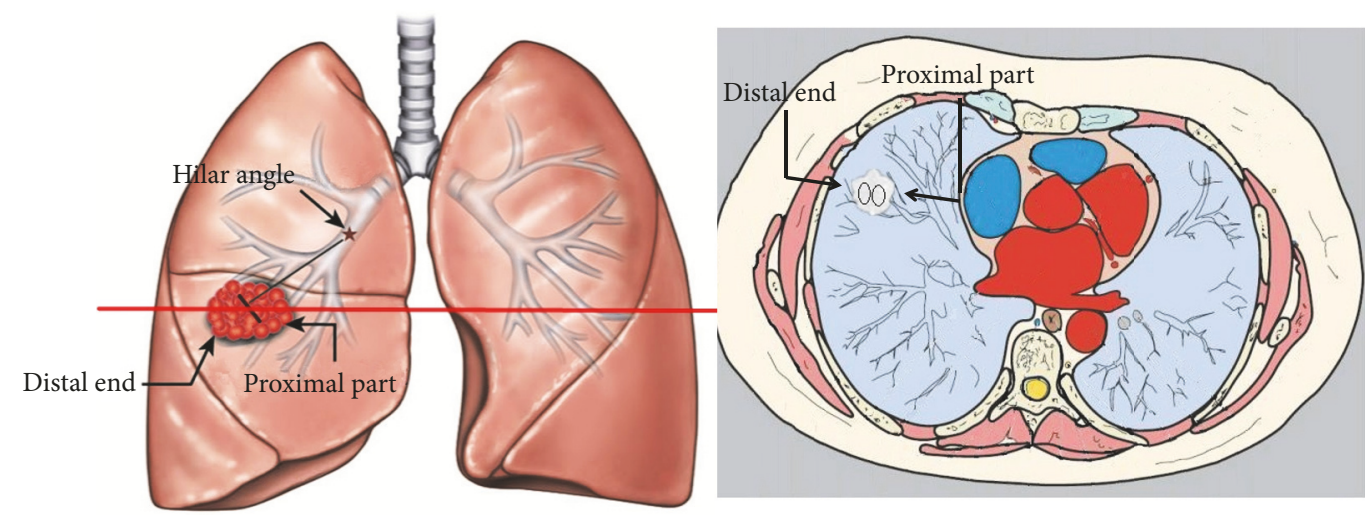

FIGURE 1: Illustration of proximal part and distal end of solitary pulmonary nodules in our study. The ipsilateral hilar angle and the direction of lung marking were the reference point.

$\mathrm{CT}$, respectively) interpreted the CT images and measured the quantitative parameters in a workstation (ADW4.6; GE Healthcare) independent of each other, and their disagreement on measurement was resolved by consensus.

We defined the area close to the ipsilateral hilar as the proximal region of SPNs and the area away from the ipsilateral hilar as the distal region (Figure 1). The region was divided along the direction of the lung marking. The iodine concentration $\left(\mathrm{IC}_{\text {les }}\right)$ in both proximal and the distal regions of SPNs on the three phases of enhanced images were measured. The chest radiologist placed a circular ROI in as large an area as possible within the SPN. All ROI measurements were measured at three successive levels and averaged. The size, shape, and position of ROIs were the same between different phases by using the copy-and-paste function. For these measurements, a circular region of interest (ROI) that avoided cavity, calcification and blood vessels were placed at specific area in SPNs, and the selected ROI on images of one phase was identical with the other two phases. The concentration of iodine $\left(\mathrm{IC}_{\mathrm{ao}}\right)$ in aorta descendens corresponding to each ROI in lesion was also measured. Normalized iodine concentrations (NIC) were calculated as the ratio of iodine concentration in lesion and aorta descendens $\left(\mathrm{NIC}=\mathrm{IC}_{\text {les }} / \mathrm{IC}_{\mathrm{ao}}\right)$, and the difference in normalized iodine concentration between the proximal and the distal regions was archived $\left(\mathrm{dNIC}=\mathrm{NIC}_{\text {pro }}-\mathrm{NIC}_{\mathrm{dis}}\right)$. Moreover, in the same slice, the mean iodine concentration (IC) in the entire region of lesion and the standard deviation (SD) of iodine concentrations were also measured. Moreover, two experienced radiologists on conventional CT images performed visual assessment.

2.5. Statistical Analysis. Commercial statistical analysis packages (version 20.0 SPSS, IBM) were used to analyze the measurements. All the data tested for normality. Mann-Whitney U-test was used to statistically compare these parameters at PP between malignant and benign nodules because data in this group did not meet the normality. The two-sample t-test was used to statistically compare these parameters at AP and VP. Receiver operating characteristic (ROC) analyses were used to compare the capability of $\mathrm{NIC}_{\text {pro }}$, $\mathrm{dNIC}$ in all three phases and visual assessment to distinguish malignant and benign SPNs. The threshold values of multiple parameters were determined by using of the ROC curves to optimize both the sensitivity and the specificity (Youden's index). $\mathrm{P}$ value $<0.05$ was considered to indicate a significant difference.

\section{Results}

Intragroup observer agreement (ICC) was used to assess the interobserver (K.T. and L.Y.W) reliability and agreement. Intragroup observer agreement (ICC) was significantly good and overall intragroup correlation coefficient in $\mathrm{NIC}_{\text {pro }}$ $\left(\mathrm{ICC}_{\text {pro }}\right)$ and $\mathrm{NIC}_{\text {dis }}\left(\mathrm{ICC}_{\text {dis }}\right)$ was 0.810 and 0.849 at PP, 0.858 and 0.786 at AP, and 0.827 and 0.796 at $\mathrm{VP}$ (all $\mathrm{P}<0.001$ ).

The data were expressed as mean $\pm \mathrm{SD}$ or median and interquartiles (P50, P25, and P75). The measurement data of $\mathrm{NIC}_{\text {pro }}, \mathrm{NIC}_{\text {dis }}$, and $\mathrm{dNIC}$ at PP, AP, and VP of all the 60 cases were summarized in Table 1 . There were significant differences in $\mathrm{NIC}_{\text {pro }}$ and $\mathrm{NIC}_{\text {dis }}$ between malignant and benign nodules in AP (mean $\mathrm{NIC}_{\text {pro }}, 0.283 \pm 0.165$ versus $0.183 \pm 0.081 ; \mathrm{P}=0.012$; mean $\mathrm{NIC}_{\text {dis }} 0.190 \pm 0.117$ versus $0.258 \pm 0.094 ; \mathrm{P}=0.024)$ and $\mathrm{VP}\left(\right.$ mean $\mathrm{NIC}_{\text {pro }}, 0.577 \pm 0.199$ versus $0.407 \pm 0.247 ; \mathrm{P}=0.005$; mean $\mathrm{NIC}_{\text {dis }} 0.406 \pm 0.142$ versus $0.591 \pm 0.247 ; \mathrm{P}=0.004)$. Significant differences were also found in $\mathrm{NIC}_{\text {pro }}$ at PP (median and interquartiles (0.67, $0.41,1.19$ versus $0.38,0.19,0.82) ; \mathrm{P}=0.037)$. However, no significant differences were found in $\mathrm{NIC}_{\text {dis }}$ at PP (median and interquartiles $(0.43,0.22,0.64$ versus $0.53,0.36,1.29)$; $\mathrm{P}=0.093$ ). Moreover, significant higher dNIC values in the proximal regions were found in malignant nodules than benign nodules at PP (median and interquartiles $(0.31,0.11$, 0.57 versus $-0.26,-0.5,-0.1) ; \mathrm{p} \leq 0.001$ ), $\mathrm{AP}$ (mean dNIC, 0.093 \pm 0.094 versus $-0.075 \pm 0.060 ; \mathrm{p} \leq 0.001$ ), and VP (mean $\mathrm{dNIC}$, $0.171 \pm 0.137$ versus $-0.183 \pm 0.127 ; \mathrm{p} \leq 0.001)$.

Quantitative assessment of IC and SD of IC at PP, AP, and VP was showed in Table 2. No differences were found in IC between malignant and benign nodules in all three phases. However, SD of IC in malignant nodules was higher than that in benign nodules at AP (malignant: 9.701 \pm 2.922 , benign: $8.245 \pm 1.796, \mathrm{P}=0.020)$. 
TABLE 1: Quantitative assessment of NIC and dNIC in the proximal and distal regions of malignant and benign nodules in PP, AP, and VP.

\begin{tabular}{lccc}
\hline Group & Malignant nodules & Benign nodules & P value \\
\hline $\mathrm{IC}_{\mathrm{PP}}$ & $11.128 \pm 6.509$ & $14.381 \pm 7.010$ & 0.089 \\
$\mathrm{IC}_{\mathrm{AP}}$ & $18.410 \pm 8.497$ & $19.520 \pm 8.675$ & 0.633 \\
$\mathrm{IC}_{\mathrm{VP}}$ & $17.440 \pm 5.637$ & $18.620 \pm 9.952$ & 0.619 \\
$\mathrm{SD}_{\mathrm{PP}}$ & $8.1723 \pm 2.176$ & $7.891 \pm 1.297$ & 0.591 \\
$\mathrm{SD}_{\mathrm{AP}}$ & $9.701 \pm 2.922$ & $8.245 \pm 1.796$ & 0.020 \\
$\mathrm{SD}_{\mathrm{VP}}$ & $8.457 \pm 2.292$ & $7.535 \pm 1.426$ & 0.060 \\
Longest diameter $(\mathrm{mm})$ & $19.26 \pm 5.999$ & $15.76 \pm 4.847$ & 0.018 \\
\hline
\end{tabular}

PP, pulmonary phase; AP, arterial phase; VP, venous phase; IC: iodine concentration; SD: standard deviation of iodine concentration in all nodules.

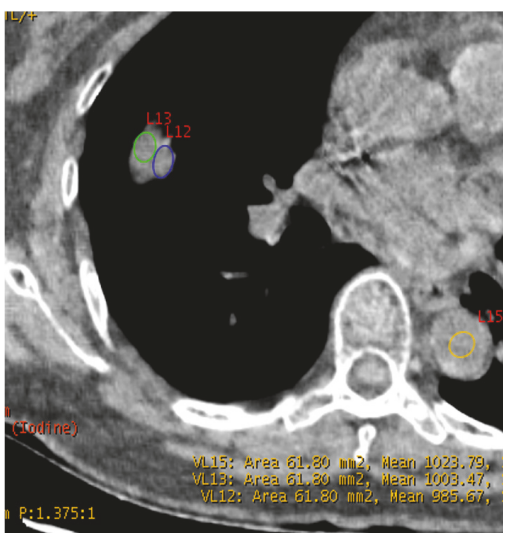

(a)

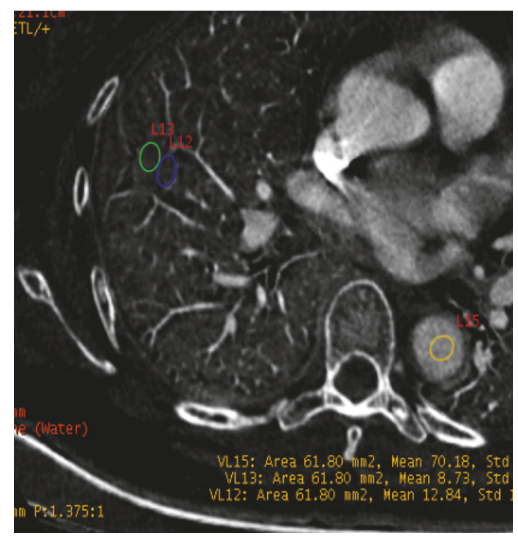

(d)

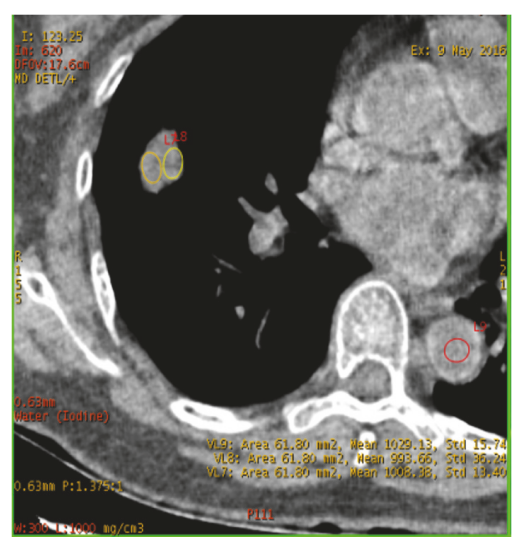

(b)

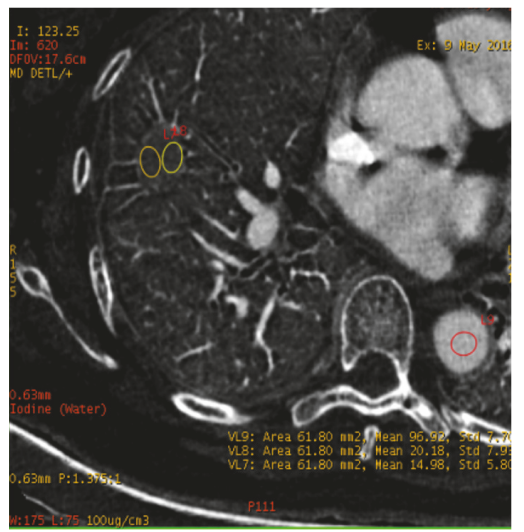

(e)

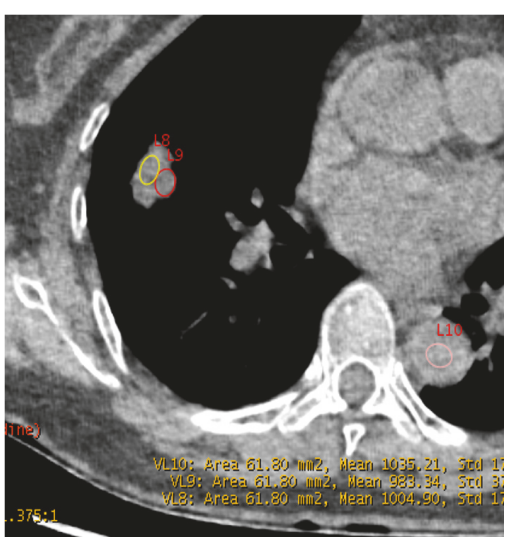

(c)

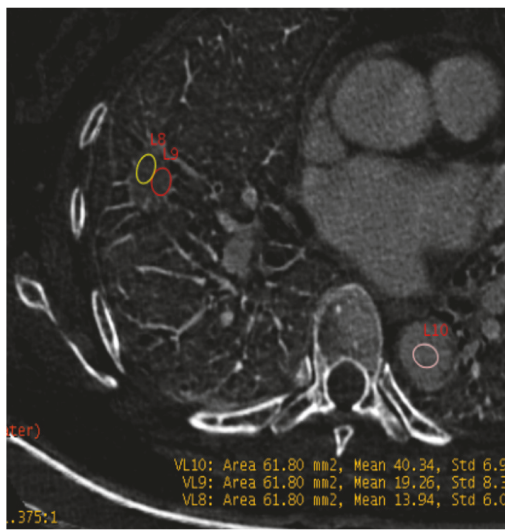

(f)

FIGURE 2: A 58-year-old woman with adenocarcinoma in the middle lobe of right lung. The spectral CT imaging (section thickness, 0.625 $\mathrm{mm}$ ) water-based material decomposition images (a-c) and iodine-based material decomposition images (d-f) at PP, AP, and VP. The region of interest (ROI) was drawn in the proximal and distal area of the lesion. $\mathrm{dNIC}(\mathrm{PP})=0.06, \mathrm{dNIC}(\mathrm{AP})=0.05$, and dNIC $(\mathrm{VP})=0.13$.

Figures 2 and 3 showed images in two patients (one with pulmonary adenocarcinoma and the other one with tuberculosis). Pseudocolor images generated from the artery phase, which visually display heterogeneity on blood supply in tumor by different colors, were showed in Figure 4 . The scatter plot of dNIC between malignant and benign nodules in three phases was displayed in Figure 5

The ROC curves for predicting malignant and benign nodules based on $\mathrm{NIC}_{\text {pro }}$ and $\mathrm{dNIC}$ in the three phases and visual assessment were shown in Figure 5. The AUC, based on NIC at VP (AUC $=0.933)$, was greater than those based on other quantitative parameters (Figure 6; Table 3). The diagnostic sensitivity and specificity of $\mathrm{NIC}_{\text {pro }}$ and $\mathrm{dNIC}$ and visual assessment were as follows: NICpro: $77 \%$ and $57 \%$ with threshold of 0.40 in PP, $67.0 \%$ and $57 \%$ with threshold of 0.47 in $\mathrm{AP}$, and $89 \%$ and $52 \%$ with threshold of 0.36 in VP; dNIC: $92 \%$ and $95 \%$ with threshold of -0.35 in PP, $89 \%$ and $90 \%$ with threshold of 0.00 in AP, and $93 \%$ and $95 \%$ with threshold of -0.07 in VP; visual assessment: $79 \%$ and $62 \%$ (Table 3). 


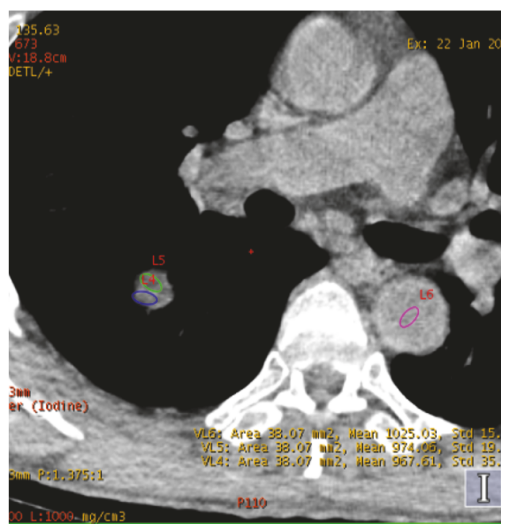

(a)

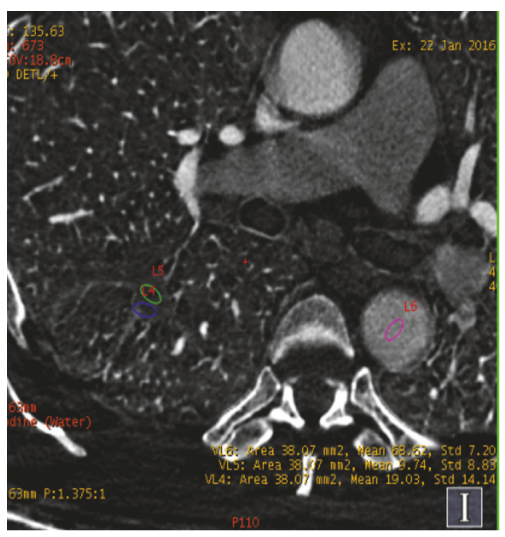

(d)

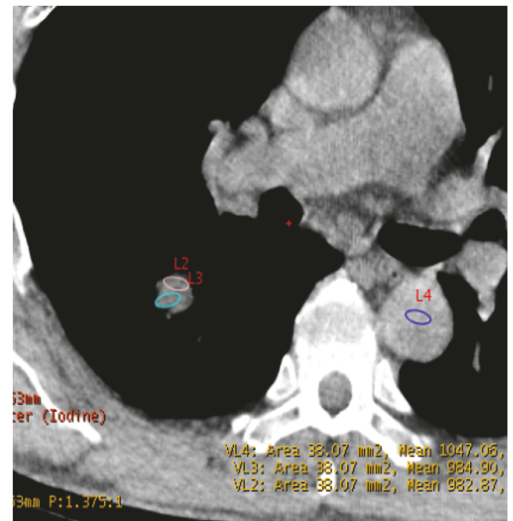

(b)

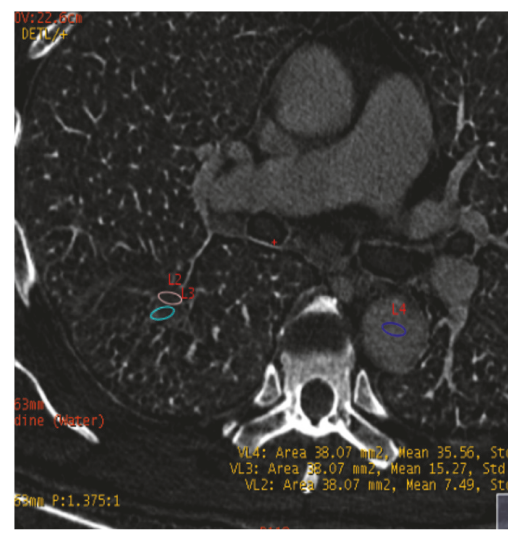

(e)

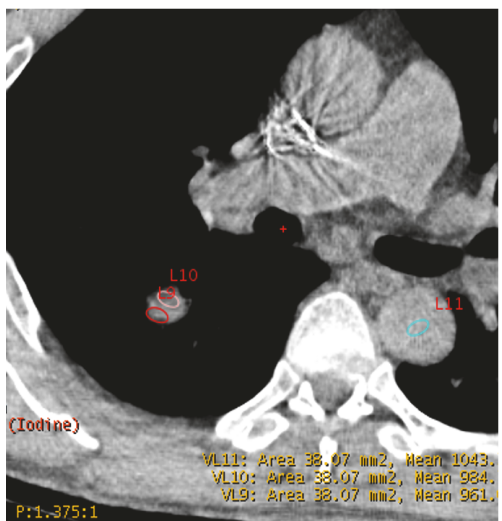

(c)

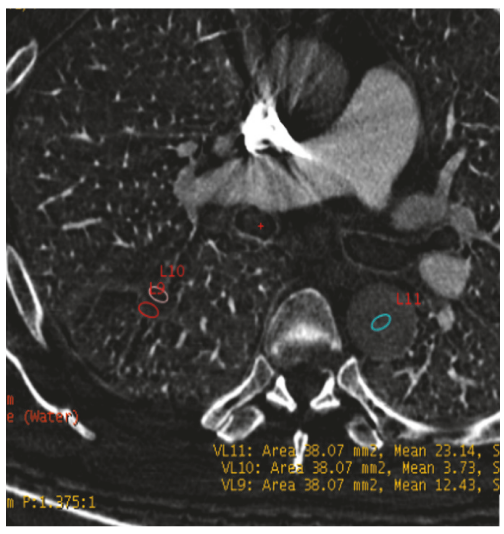

(f)

Figure 3: A 60-year-old man with tuberculosis in the inferior lobe of right lung. The spectral CT (section thickness, $0.625 \mathrm{~mm}$ ) water-based material decomposition images (a-c) and iodine-based material decomposition image (d-f) at PP, AP, and VP. The region of interest (ROI) was drawn in the proximal and distal area of the lesion. $\mathrm{dNIC}(\mathrm{PP})=-0.38$, dNIC $(\mathrm{AP})=-0.14$, and dNIC $(\mathrm{VP})=-0.22$.

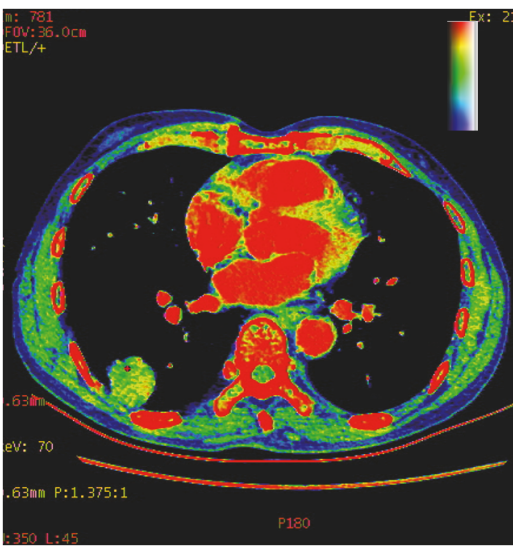

(a)

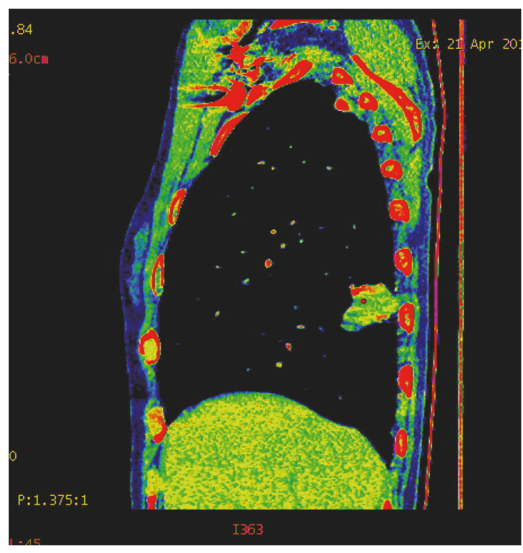

(b)

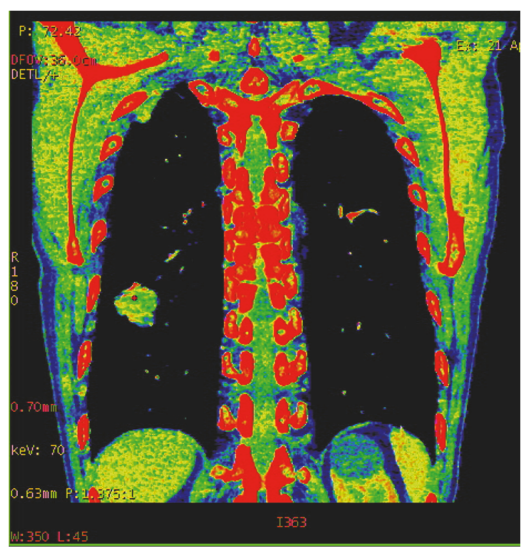

(c)

FIGURE 4: An 80-year-old man with adenocarcinoma in the inferior lobe of right lung. Pseudocolor images of spectral CT on the artery phase can visually display distribution difference of iodine concentration based on blood supply in tumor by different colors. The color in proximal region was brighter than that of distal one. 
TABLE 2: Quantitative assessment of IC and standard deviation of iodine concentration in PP, AP, and VP.

\begin{tabular}{lccc}
\hline Group & NIC $_{\text {pro }}$ & NIC $_{\text {dis }}$ & dNIC \\
\hline PP & & & $(0.31,0.11,0.57)$ \\
$\quad$ Malignant nodules & $(0.67,0.41,1.19)$ & $(0.43,0.22,0.64)$ & $(-0.26,-0.5,-0.1)$ \\
Benign nodules & $(0.38,0.19,0.82)$ & $(0.53,0.36,1.29)$ & $\mathrm{p} \leq 0.001$ \\
$\quad$ P value & 0.037 & 0.093 & $0.093 \pm 0.094$ \\
AP & & & $-0.075 \pm 0.060$ \\
Malignant nodules & $0.283 \pm 0.165$ & $0.190 \pm 0.117$ & $\mathrm{p} \leq 0.001$ \\
Benign nodules & $0.183 \pm 0.081$ & $0.258 \pm 0.094$ & 0.024 \\
P value & 0.012 & & $-0.171 \pm 0.137$ \\
VP & & $0.406 \pm 0.142$ & $\mathrm{p} \leq 0.001$ \\
Malignant nodules & $0.577 \pm 0.199$ & $0.591 \pm 0.247$ & 0.004 \\
Benign nodules & $0.407 \pm 0.247$ & 0.005 & \\
P value & & & \\
\hline
\end{tabular}

PP, pulmonary phase; AP, arterial phase; VP, venous phase; $\mathrm{NIC}_{\text {pro }}$, normalized iodine concentration in the proximal region; $\mathrm{NIC}_{\mathrm{dis}}$, normalized iodine concentration in the distal region; dNIC, $\mathrm{NIC}_{\mathrm{pro}}-\mathrm{NIC}_{\mathrm{dis}}$.

TABLE 3: Diagnostic indices of NICpro, dNIC in PP, AP, and VP, and other methods for differentiating malignant and benign SPNs.

\begin{tabular}{|c|c|c|c|c|c|c|c|c|c|}
\hline Criteria & AUC & $\begin{array}{c}\text { Sensitivity } \\
(\%)\end{array}$ & $\begin{array}{c}\text { Specificity } \\
(\%)\end{array}$ & $\mathrm{TP}$ & $\mathrm{FP}$ & FN & $\mathrm{TN}$ & $\begin{array}{l}\text { PPV } \\
(\%)\end{array}$ & NPV (\%) \\
\hline NICpro(PP) $>0.40$ & 0.644 & 77 & 57 & 26 & 13 & 13 & 8 & 67 & 38 \\
\hline NICpro(AP) $>0.47$ & 0.716 & 67 & 57 & 3 & 0 & 36 & 21 & 100 & 37 \\
\hline NICpro(VP) $>0.36$ & 0.714 & 89 & 52 & 35 & 10 & 4 & 11 & 78 & 73 \\
\hline $\mathrm{dNIC}(\mathrm{PP})>-0.35$ & 0.923 & 92 & 95 & 37 & 13 & 1 & 9 & 74 & 90 \\
\hline $\mathrm{dNIC}(\mathrm{AP})>0.00$ & 0.924 & 89 & 90 & 35 & 2 & 4 & 19 & 94 & 82 \\
\hline $\mathrm{dNIC}(\mathrm{VP})>-0.07$ & 0.933 & 93 & 95 & 37 & 1 & 2 & 20 & 97 & 90 \\
\hline Visual assessment (conventional CT) & 0.707 & 79 & 62 & 31 & 8 & 8 & 13 & 79 & 62 \\
\hline
\end{tabular}

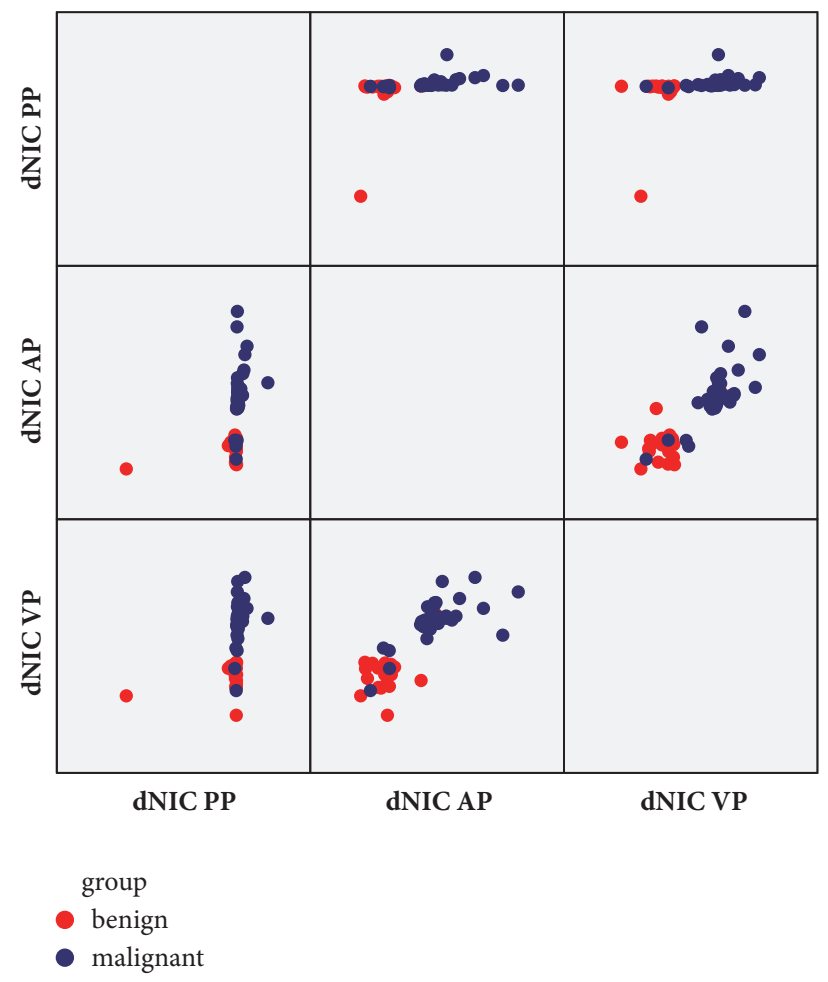

Figure 5: The three-dimensional scatter plots of dNIC between malignant and benign nodules in three phases. From the scatter plot, the dNIC distribution of malignant and benign nodules reflects distribution difference of iodine concentration.

\section{Discussion}

Nowadays, the detection rate of SPN can be up to $51 \%$ with the widespread use of multidetector CT (MDCT). Most of the detected SPNs have benign causes; however, there are still $20-30 \%$ of them which are malignant, which are mainly in the early stage of lung cancer [24]. Lung cancer, the leading cause of cancer death throughout the world, is responsible for an estimated 158,040 deaths in 2015 [25]. Overall 5year survival rates in patients with lung cancer remains rather low at $15 \%$; however, stage IA of lung cancer that is treated with resection may portend a long-term survival of $80 \%$ [24]. Therefore, it is essential of the differentiation and treatment for the pulmonary nodules of strongly suspicious malignancy. However, it remains challenge of differential diagnosis because of the overlap in imaging features between malignant and benign nodules. Hence, the methodology of imaging has been developing from traditional morphological examinations to dynamic functional examinations.

Different techniques to obtain dual-energy data have been proposed and pursued by various vendors: rapid $\mathrm{kVp}$ switching (GE Healthcare, Milwaukee, WI); energy-sensitive sandwich detectors (Philips Medical Systems, Cleveland, $\mathrm{OH}$ ); and dual-source CT (Siemens Healthcare, Forchheim, Germany). The system in our study was rapid $\mathrm{kVp}$ switching (GE Healthcare, Milwaukee, WI). Spectral CT can realize rapid alternation (in the order of 1000 times per second) of the tube potentials between, for instance, 80 and $140 \mathrm{kv}$ during the gantry rotation $[10,26]$. At present, dual-energy 


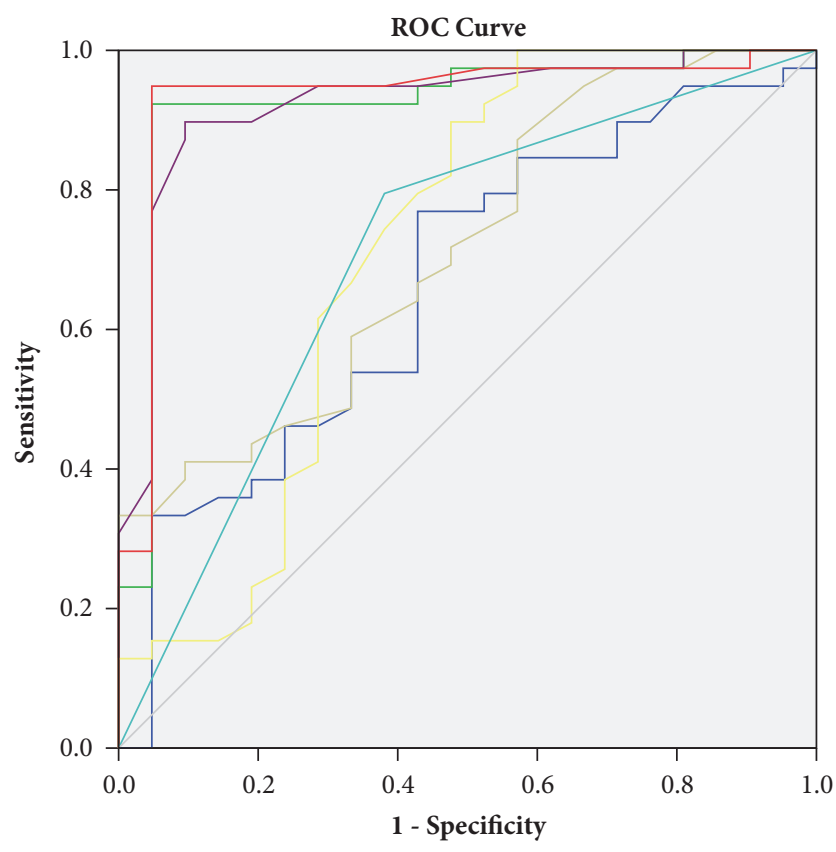

Diagonal segments are produced by ties.

$\begin{array}{ll}\text { Source of the Curve } & \\ \text { - NICpro PP } & \text { NICpro VP } \\ \text { - dNIC PP } & - \text { dNIC VP } \\ \text { NICpro AP } & - \text { Visual } \\ \text { - dNIC AP } & - \text { Reference Line }\end{array}$

FIGURE 6: Receiver operating characteristic (ROC) curves by using NIC in the proximal region and dNIC to differentiate lung cancers from benign nodules during pulmonary arterial phase (PP), arterial phase (AP), and venous phase (VP). dNIC, the NIC value difference between the proximal and distal regions of nodules; NIC, normalized iodine concentration; ROC, receiver operating characteristic.

CT equipment used in clinical practice is spectral CT and dual-source CT. As a functional imaging method, spectral $\mathrm{CT}$ provides multiple parameters by a rapid $\mathrm{kVp}$ switching technique, such as material decomposition imaging, and it has the potential value of application in neoplastic diseases [11-13, 27].

According to our research, iodine concentrations in malignant nodules were lower than that in benign ones. The reasons may be that in the benign nodules, inflammatory nodules $(n=15$, including 11 with focal-organizing pneumonia, 2 with granulomatous inflammation, and 2 with pulmonary inflammation) account for about $71 \%$ (15/21) in our research. In previous study, patients with inflammatory masses also had significantly higher normalized iodine concentration values than patients with lung cancers. Because granulomatous inflammation and organizing pneumonia are formed by proliferation of inflammatory granulation tissue or residual of acute inflammation, the rich and dilatate capillaries of masses are stimulated by inflammation gradually [28]. The earlier studies had also shown that iodine concentration of spectral CT was positively correlated with the expression of VEGF in NSCLC [29]. In the spectral imaging, iodine and water were selected as the basis pair for material decomposition image, because that iodine, being the main ingredient of contrast medium, directly reflects the number of supplying vessels and blood flow in the SPNs $[14,28,29]$.

Our results against spectral CT were consistent with the study of Zhao et al. against PET/CT [18]. There were significant differences in the NIC between malignant and benign SPNs, with respect to both of the proximal and distal region at $\mathrm{AP}$ and $\mathrm{VP}$. In addition, the NIC of malignant SPNs was significantly higher in the proximal regions than that in the distal regions. Based on pathologic analysis, these observations might be associated with blood supply of SPN. As we all know, cancer tends to grow towards the blood vessels to find nutrition [30]; thus the proximal region of malignant nodules was usually rich in blood supply. After enhanced CT scan, high-density iodine is the main media material in the blood vessel, so the iodine concentration in the ROI directly reflects its blood supply. However, no significant differences were found in iodine concentration between malignancy and benignancy (malignant versus benign: $\mathrm{P}$ $=0.089,0.633,0.619$, respectively, in $\mathrm{PP}, \mathrm{AP}$, and VP). In addition, our results clearly showed that the $\mathrm{SD}$ of iodine concentration of malignant nodules was higher than that of benign ones. As far as we know, malignant tumors showed much more inhomogeneous enhancement than benign ones. The establishment of angiogenesis and vascular networks usually occur in malignant tumor, which could not support the rapid growth of neoplasm, and the subsequent reduction in the delivery of oxygen renders lung cancer hypoxic. Moreover, the difference of SD was statistically significant in VP $(\mathrm{P}=0.020)$. In other words, the spectral CT in VP can better show the distribution difference of iodine concentration of SPN by SD of iodine concentrations.

The present study has some limitations. First, the study population was relatively small in our study, necessitating larger prospective studies for confirmation. Second, volumetric measurements of the iodine concentration should be measured in study. Instead of volumetric measurement, all ROI measurements were measured at three successive levels and averaged in our research. Further clinical trials need to be performed in the future. Last, data could be limited to the single-source platform used and may not be applicable to the dual-source CT system. As we know, both rapid kilovoltage switching single-source spectral CT and detector-based dualsource CT can perform two-material decomposition analysis such as iodine- and water-based decomposition from high- and low-energy projection [31]. On the dual-source CT system, it is quite expected that the data will also be applicable for dual-source CT, but it need further study to confirm.

In conclusion, the difference of iodine concentration spatial distribution related to blood supply in SPNs helps for differentiation of malignancy and benignancy on contrastenhanced spectral CT by analyzing multiple quantification parameters. In addition, SD of iodine concentrations in venous phase was found to reflect distribution difference of iodine concentration better. Quantitative analysis of iodine concentration spatial distribution difference based on blood supply by spectral CT may be a promising new method for 
differentiating malignant and benign pulmonary nodules and has the potential application values in future.

\section{Data Availability}

The Excel data used to support the findings of this study are available from the corresponding author upon request.

\section{Conflicts of Interest}

The authors declare that there are no conflicts of interest regarding the publication of this paper.

\section{Acknowledgments}

This study has received funding by Zhejiang Provincial Natural Science Foundation of China (Grant NO. Y17H180046), Grant Recipient: Xiangwu Zheng.

\section{References}

[1] D. Ost, A. M. Fein, and S. H. Feinsilver, "The solitary pulmonary nodule," The New England Journal of Medicine, vol. 348, no. 25, pp. 2535-2542, 2003.

[2] A. Snoeckx, P. Reyntiens, D. Desbuquoit et al., "Evaluation of the solitary pulmonary nodule: size matters, but do not ignore the power of morphology," Insights into Imaging, vol. 9, no. 1, pp. 73-86, 2018.

[3] A. M. Maffione, G. Grassetto, L. Rampin et al., "Molecular imaging of pulmonary nodules," American Journal of Roentgenology, vol. 202, no. 3, pp. W217-W223, 2014.

[4] X.-D. Ye, J.-D. Ye, Z. Yuan, W.-T. Li, and X.-S. Xiao, "Dynamic CT of solitary pulmonary nodules: Comparison of contrast medium distribution characteristic of malignant and benign lesions," Clinical and Translational Oncology, vol. 16, no. 1, pp. 49-56, 2014.

[5] X. Wang, L. Lv, Q. Zheng, X. Huang, and B. Li, “Differential diagnostic value of 64-slice spiral computed tomography in solitary pulmonary nodule," Experimental and Therapeutic Medicine, vol. 15, no. 6, pp. 4703-4708, 2018.

[6] S. J. Swensen, R. W. Viggiano, D. E. Midthun et al., "Lung nodule enhancement at CT: Multicenter study," Radiology, vol. 214, no. 1, pp. 73-80, 2000.

[7] J. J. Erasmus, J. E. Connolly, H. P. McAdams, and V. L. Roggli, "Solitary pulmonary nodules: part I. Morphologic evaluation for differentiation of benign and malignant lesions," RadioGraphics, vol. 20, no. 1, pp. 43-58, 2000.

[8] M. Karçaaltincaba and A. Aktas, "Dual-energy CT revisited with multidetector CT: review of principles and clinical applications," [J]. Diagnostic Interventional Radiology, vol. 17, no. 3, pp. 181-94, 2010.

[9] T. Henzler, J. Shi, and H. Jafarov, "Functional CT imaging techniques for the assessment of angiogenesis in lung cancer," Transl Lung Cancer Res, vol. 1, no. 1, pp. 78-83, 2012.

[10] D. Simons, M. Kachelrieß, and H.-P. Schlemmer, "Recent developments of dual-energy CT in oncology," European Radiology, vol. 24, no. 4, pp. 930-939, 2014.
[11] H. Xiao, Y. Liu, H. Tan et al., "A pilot study using low-dose Spectral CT and ASIR (Adaptive Statistical Iterative Reconstruction) algorithm to diagnose solitary pulmonary nodules," BMC Medical Imaging, vol. 15, no. 1, 2015.

[12] Y. Zhang, J. Tang, J. Xu, J. Cheng, and H. Wu, "Analysis of pulmonary pure ground-glass nodule in enhanced dual energy CT imaging for predicting invasive adenocarcinoma: Comparing with conventional thin-section CT imaging," Journal of Thoracic Disease, vol. 9, no. 12, pp. 4967-4978, 2017.

[13] G. Liu, M. Li, G. Li et al., "Assessing the blood supply status of the focal ground-glass opacity in lungs using spectral computed tomography," Korean Journal of Radiology, vol. 19, no. 1, pp. 130138, 2018.

[14] Y. Zhang, J. Cheng, X. Hua et al., "Can Spectral CT Imaging Improve the Differentiation between Malignant and Benign Solitary Pulmonary Nodules?" PLoS ONE, vol. 11, no. 2, p. e0147537, 2016.

[15] X. Meng, C. Ni, Y. Shen et al., "Differentiating malignant from benign gastric mucosal lesions with quantitative analysis in dual energy spectral computed tomography," Medicine, vol. 96, no. 2, p. e5878, 2017.

[16] X.-H. Chen, K. Ren, P. Liang, Y.-R. Chai, K.-S. Chen, and J.B. Gao, "Spectral computed tomography in advanced gastric cancer: Can iodine concentration non-invasively assess angiogenesis?" World Journal of Gastroenterology, vol. 23, no. 9, pp. 1666-1675, 2017.

[17] P. Lv, X. Z. Lin, J. Li, W. Li, and K. Chen, “Differentiation of small hepatic hemangioma from small hepatocellular carcinoma: Recently introduced spectral CT method," Radiology, vol. 259, no. 3, pp. 720-729, 2011.

[18] L. Zhao, L. Tong, J. Lin et al., "Characterization of solitary pulmonary nodules with $18 \mathrm{~F}-\mathrm{FDG}$ PET/CT relative activity distribution analysis," European Radiology, vol. 25, no. 7, pp. 1837-1844, 2015.

[19] O. Schillaci and F. F. Calabria, "Comments on characterization of solitary pulmonary nodules with 18F-FDG PET/CT relative activity distribution analysis," Journal of Thoracic Disease, vol. 7, no. 10, pp. 1708-1712, 2015.

[20] A. M. García Vicente, V. M. Pérez-García, and Á. Soriano Castrejón, " ${ }^{18}$ F-fluorodeoxyglucose positron emission tomography/computed tomography characterization of solitary pulmonary nodules: Can we do better?" Journal of Thoracic Disease, vol. 7, no. 8, pp. E215-E218, 2015.

[21] W. Li, H. Pang, Q. Liu, and J. Zhou, "The role of ${ }^{18}$ F-FDG PET or ${ }^{18}$ F-FDG-PET/CT in the evaluation of solitary pulmonary nodules," European Journal of Radiology, vol. 84, no. 10, pp. 2032-2037, 2015.

[22] G. Schmid-Bindert, T. Henzler, T. Q. Chu et al., "Functional imaging of lung cancer using dual energy CT: How does iodine related attenuation correlate with standardized uptake value of 18FDG-PET-CT?” European Radiology, vol. 22, no. 1, pp. 93-103, 2012.

[23] M. T. Truong, J. P. Ko, S. E. Rossi et al., "Update in the evaluation of the solitary pulmonary nodule," RadioGraphics, vol. 34, no. 6, pp. 1658-1679, 2014.

[24] A. M. Furman, J. Z. Dit Yafawi, and A. O. Soubani, "An update on the evaluation and management of small pulmonary nodules," Future Oncology, vol. 9, no. 6, pp. 855-865, 2013.

[25] R. L. Siegel, K. D. Miller, and A. Jemal, "Cancer statistics, 2015," CA: A Cancer Journal for Clinicians, vol. 65, no. 1, pp. 5-29, 2015. 
[26] J. Fornaro, S. Leschka, D. Hibbeln et al., "Dual- and multienergy CT: approach to functional imaging," Insights into Imaging, vol. 2, no. 2, pp. 149-159, 2011.

[27] T. W. Cramer, J. G. Fletcher, R. G. Paden et al., "A primer on the use of dual-energy CT in the evaluation of commonly encountered neoplasms," Abdominal Radiology, vol. 41, no. 8, pp. 1618-1631, 2016.

[28] W. S. Hou, H. W. Wu, Y. Yin, J. J. Cheng, Q. Zhang, and J. R. Xu, "Differentiation of lung cancers from inflammatory masses with dual-energy spectral CT imaging," Academic Radiology, vol. 22, no. 3, pp. 337-344, 2015.

[29] G. J. Li, J. Gao, G. L. Wang, C. Q. Zhang, H. Shi, and K. Deng, "Correlation between vascular endothelial growth factor and quantitative dual-energy spectral CT in non-small-cell lung cancer," Clinical Radiology, vol. 71, no. 4, pp. 363-368, 2016.

[30] P.-H. Wu, A. Giri, S. X. Sun, and D. Wirtz, "Three-dimensional cell migration does not follow a random walk," Proceedings of the National Acadamy of Sciences of the United States of America, vol. 111, no. 11, pp. 3949-3954, 2014.

[31] M. Patino, A. Prochowski, M. D. Agrawal et al., "Material separation using dual-energy CT: Current and emerging applications," RadioGraphics, vol. 36, no. 4, pp. 1087-1105, 2016. 


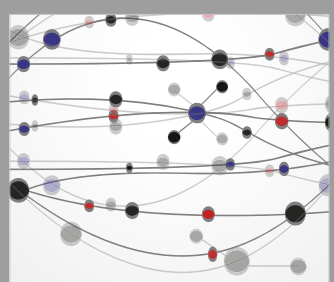

The Scientific World Journal
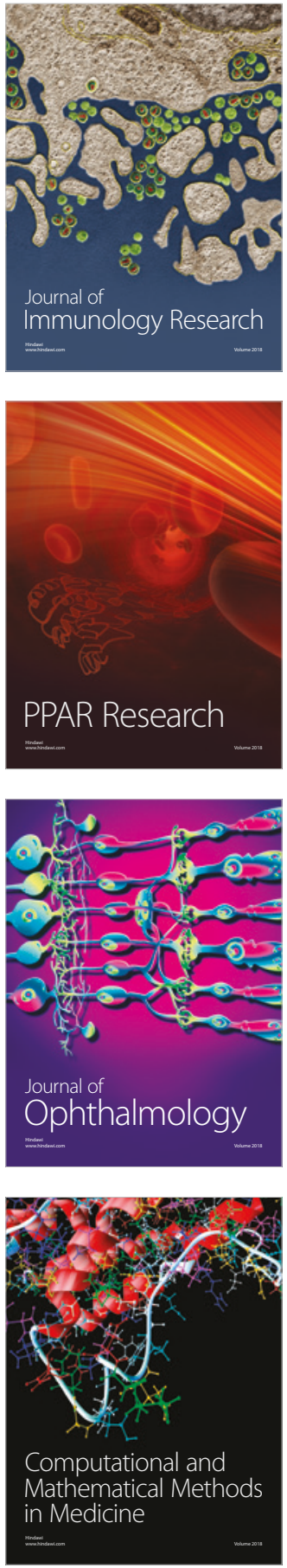

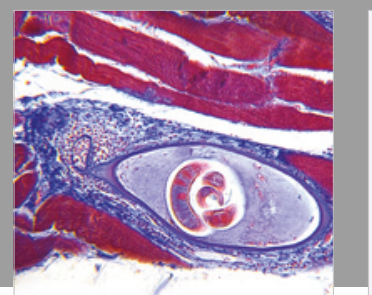

Gastroenterology Research and Practice

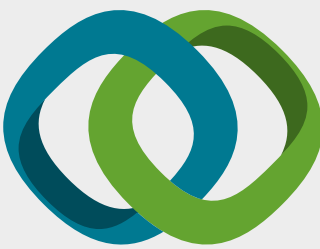

\section{Hindawi}

Submit your manuscripts at

www.hindawi.com
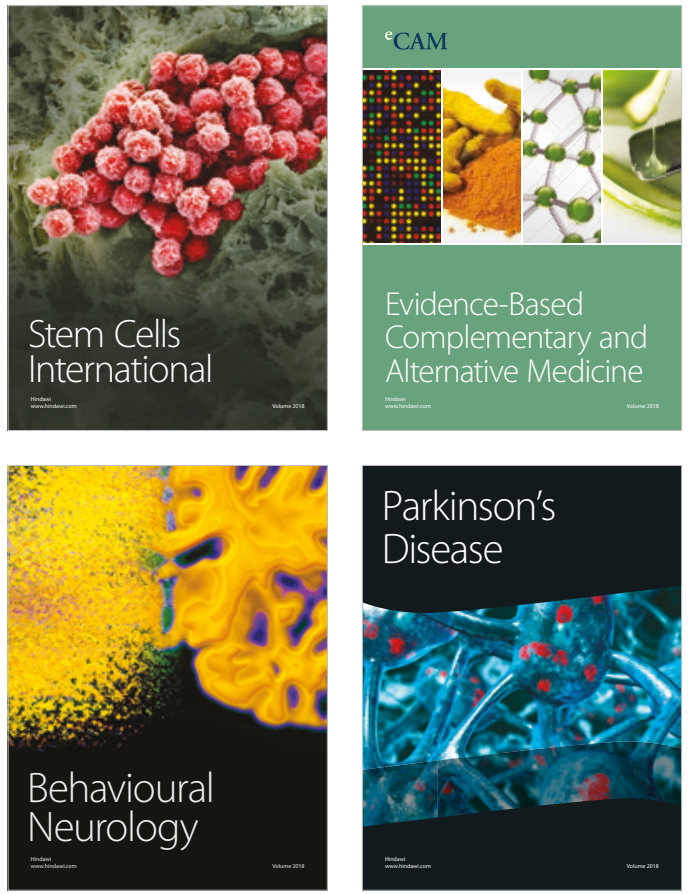

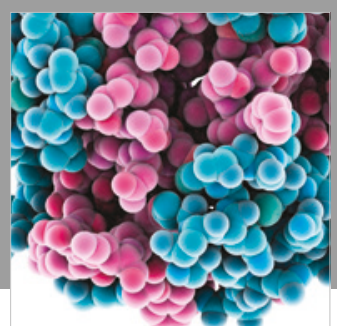

ournal of

Diabetes Research

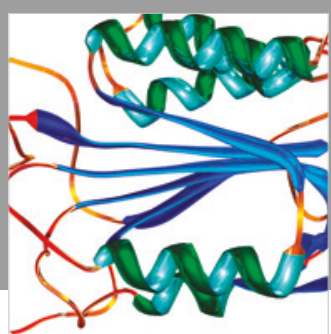

Disease Markers
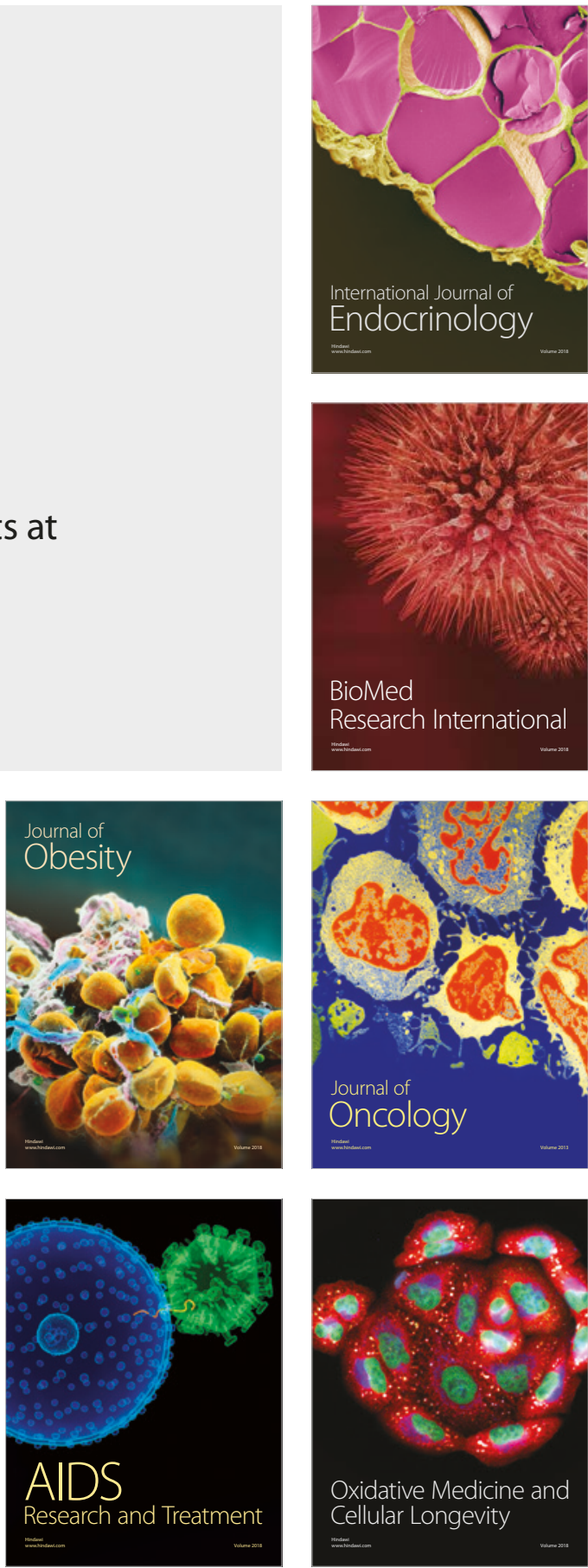